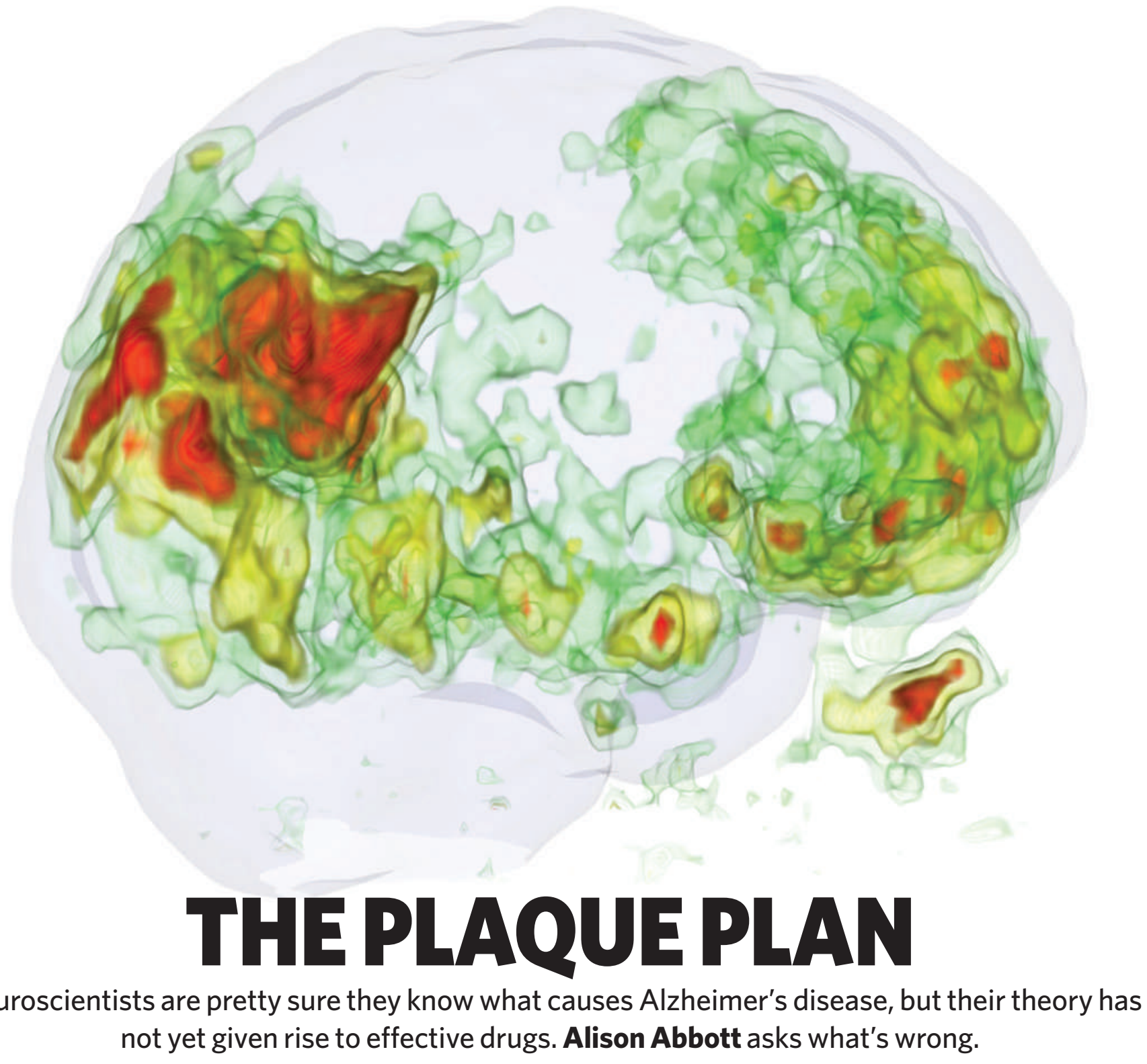

$\mathrm{t}$ is sad and bewildering to watch a parent slowly fade away, the years of authority dissolving into childlike moodiness. But it is an experience that more and more people will face as the number of elderly people with Alzheimer's disease climbs to the 80 million or so expected by 2040 (ref. 1).

If a strong hypothesis were enough to help, then treatments should be on the shelf. For years researchers have been confident that the disease is caused by protein 'plaques' and 'tangles' that eat away at the brain - and pharmaceutical companies have spent untold millions identifying and testing therapies that interfere with these structures.

But medical need and mechanism are not, it seems, enough. It has come as an acute disappointment that the first clinical trials of therapies designed to deal with the plaques have failed to help patients. In July this year, participants at the annual Alzheimer's Association meeting in Chicago, Illinois, heard that two major trials, one a phase III including 1,600 people, the other a phase II involving 234 people, failed to improve the mental state of patients even though they had looked promising in earlier studies. Another phase III trial was abandoned in 2007 when it was similarly unsuccessful. Something seems in need of a rethink, but what? The presumed biological mechanism - or the trials themselves?

Researchers say that they remain confident about their hypothesis. Their concerns lie in the way that the trials have been done. Because diagnosis is relatively rudimentary, some of the recruited patients may have had a different sort of dementia from Alzheimer's, and all of them may have had such advanced disease that their brains were irreversibly damaged. "We diagnose people when they already show dementia, not at a more helpful earlier stage," says Bruno Dubois, a neurologist at the Hôpital de la Salpêtrière in Paris. "It is like diagnosing someone with
Parkinson's disease when they are bedridden, not when they develop a tremor." This means that the trial results are not so much negative as uninterpretable.

Researchers and pharmaceutical companies had anticipated these problems, but hoped that "It is like diagnosing the trials would show some someone with Parkinson's when they are bedridden, not when they develop a tremor."

- Bruno Dubois therapeutic effect anyway. They pushed ahead because of the pressing medical need and the potential therapeutic market. At the same time, scientists have started work to improve the next round of trials by launching major international research programmes to identify biological markers of disease progression, such as those that expose plaques in living brains (pictured above). Such biomarkers might eventually reveal invisible equivalents to the Parkinson's tremor so that the disease can be recognized before dementia sets in and so that researchers can observe directly whether a 
drug is doing the biological work expected of it. Researchers are already incorporating the most promising biomarkers into many of the clinical trials now under way.

Only with biomarkers in hand will scientists be able to seriously test the theory of Alzheimer's on which they have staked so much. "The theory is not proven, and we have to be open to the fact that it could be wrong," says Paul Aisen, a neurologist at the University of California, San Diego, who runs the US Alzheimer's Disease Co-operative Study for the National Institute of Aging in La Jolla, California. "But the trials so far have simply not tested the hypothesis."

\section{Deep roots}

The hypothesis in question has roots that can be traced back more than a century to Alois Alzheimer, a physician and neuropathologist in Munich, Germany, who followed the five-year mental decline of one of his patients, Auguste Deter. When Deter died in 1906, Alzheimer scrutinized her brain using a staining technique developed by his colleague Franz Nissl. He saw a mess of clumped proteins in the form of plaques, and tangles of fibrils like snarledup thread. These characteristic features, which until recently could be seen only at autopsy, still provide the most secure diagnosis for the disease, together with neuropsychiatric assessments of memory, language and other cognitive functions.

When molecular biologists started to dissect the structures that Alzheimer observed, they found that plaques are made up of small, sticky protein fragments, or peptides. These peptides, the most common of which is called $\beta$-amyloid, are cleaved from a larger amyloid precursor protein (APP) by the enzymes $\beta$-secretase and $\gamma$-secretase. Neurofibrillary tangles, inside neurons, are now known to be made of an insoluble protein called tau. The prevailing theory holds that Alzheimer's disease is initiated when APP - the normal function of which is unknown - is converted to toxic amyloid peptides that are taken up and released by plaques. This stresses cells in a way that promotes so much phosphorylation of tau that it forms tangles. The process is known as the amyloid cascade. As the plaques and tangles spread from the hippocampus into the cerebral cortex, the people affected gradually lose their memory, their logic and eventually the ability to speak and move. In this weakened state, patients typically die of pneumonia.

The only drugs available for Alzheimer's patients aim to treat symptoms, trying to chemically stimulate areas of the brain that are already damaged by the time dementia sets in. They are marginally effective at best. So in the past five years, academic groups and pharmaceutical companies have focused on preventing the plaques and tangles from forming, or dismantling those already there.

So far, these strategies have generated a string of failures. Aisen was principal investigator on the North American trial of a drug called tramiprosate (Alzhemed) being developed by Neurochem, a biopharmaceutical company based in Montreal, Canada. It was the first trial based on the amyloid-cascade hypothesis to reach this advanced stage of testing and as such was greatly anticipated by the community. Tramiprosate is a small chemical that inhibits the formation of plaques in animal tests by binding to $\beta$-amyloid. Aisen's team followed up more than 1,000 patients for longer than 18 months but did not detect any change to their mental states and Neurochem has since pulled out of further clinical testing.

The two trials that failed this year used different approaches to attack amyloid, but with no greater success. Myriad Genetics' compound tarenflurbil (Flurizan) was designed to prevent the build-up of amyloid plaques by inhibiting $\gamma$-secretase. Myriad says that the drug might not have reached appropriate concentrations in the brain and the company abandoned it when it failed to show benefit. Elan Pharmaceuticals' bapineuzumab, a humanized monoclonal antibody that binds directly to $\beta$-amyloid and mimics an immune system attack on plaques, fared little better.

Perhaps the cruellest blow came when Clive Holmes of the Moorgreen Hospital in Southampton, UK, reported this July ${ }^{2}$ on patients six years after they received an experimental amyloid peptide vaccine developed by Elan. The vaccine was designed to teach the immune

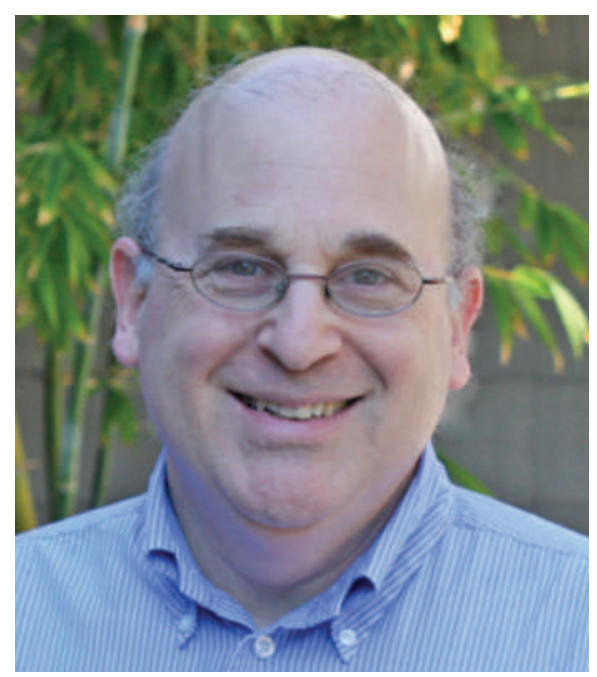

Paul Aisen suspects that some patients in Alzheimer's trials have other types of dementia. system to recognize amyloid as being foreign so that it would launch an attack against the protein. Post-mortem analyses showed that almost all the patients had stripped-down amyloid plaques, despite most of them having progressed to severe dementia before they died.

Some researchers were left wondering whether too much emphasis had been placed on removing the plaques. "It was only a small number of patients," says Karen Duff, an Alzheimer's researcher at Columbia University in New York, "but one has to question the amyloid-cascade theory, which dictates that clearance of the plaques should be correlated with disease improvement." She notes that tau tangles were still present in the autopsied brains. "This, for me, showed that far too much attention has been given to amyloid proteins, whereas other targets such as tau itself and other pathological features have been neglected."

\section{Protein principles}

Researchers have very strong reasons for believing that plaques and tangles are the cause of Alzheimer's symptoms, rather than just markers of it. Mutated versions of APP can cause an early-onset, hereditary form of the disease in which amyloid peptide has a greater propensity to accumulate in the brain years before symptoms develop ${ }^{3}$. Mice engineered to produce mutant APP that increases production of $\beta$-amyloid develop Alzheimer's-like symptoms that can be reversed by reducing tau expression ${ }^{4}$.

And even if plaques and tangles do cause the symptoms, it does not mean that removing plaques and tangles will treat them. These structures may damage the brain in ways that cannot be repaired by simply dissolving the offending protein accumulations. "It could well be that there has been too much focus on early events of the amyloid cascade," admits Simon Lovestone, a psychiatrist and neuroscientist at London's Institute of Psychiatry. "But let's not throw the baby out with the bath water there is just too much convincing preclinical research that supports the amyloid-cascade hypothesis."

Rather than rethinking the hypothesis, researchers are rethinking the trials. Aisen says that a major problem for the tramiprosate trial "was the huge variability in rates of decline among patients". In retrospect, he estimates that one-fifth of the patients recruited might not have had Alzheimer's at all, and so could not have been helped by therapy geared to disrupt the amyloid build-up typical of the disease. Only $50-70 \%$ of dementia cases are 


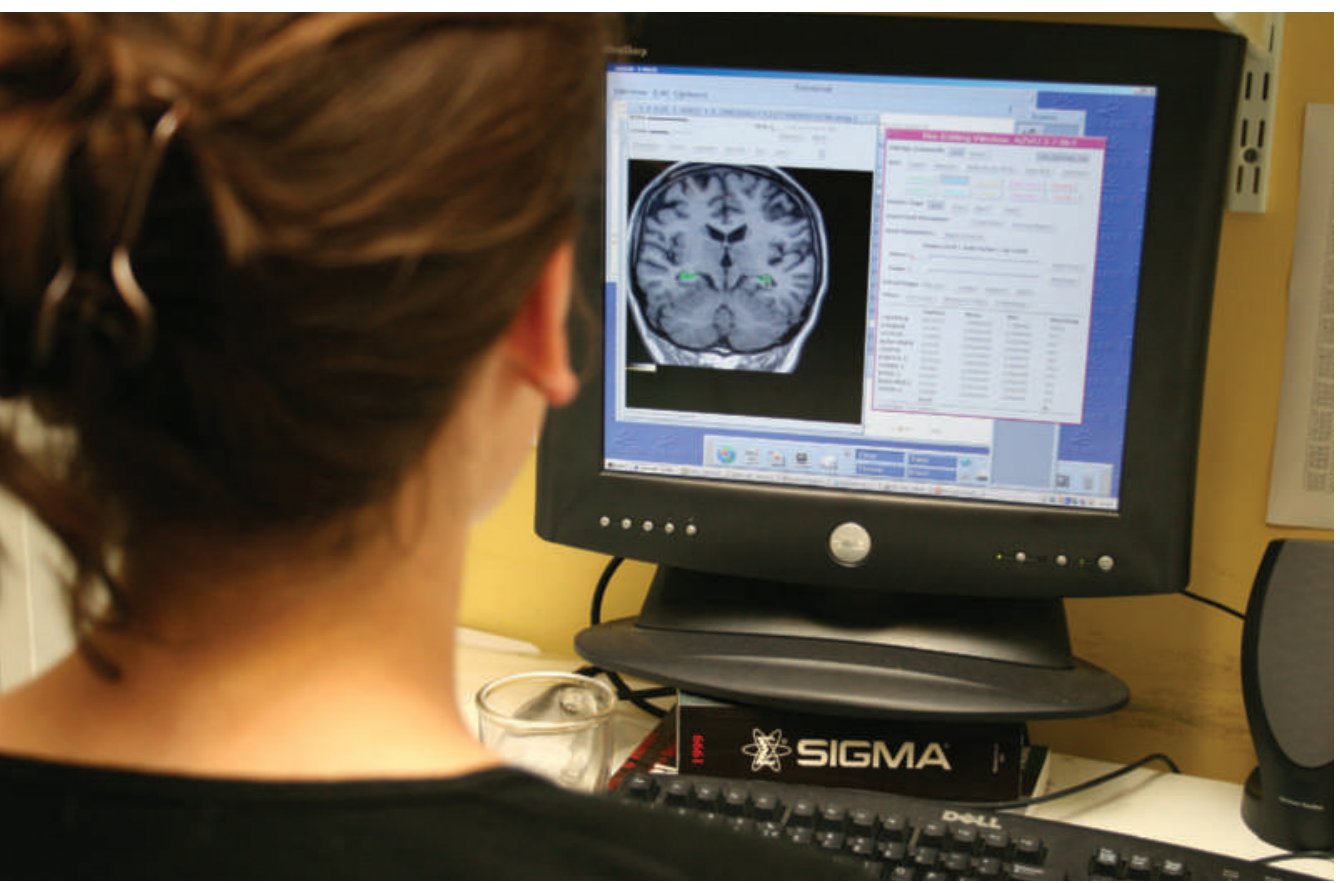

The volume of the brain's hippocampus may serve as a marker of Alzheimer's progression.

the Alzheimer's type; the rest have dementias that have other pathological indicators and neuropsychiatric profiles.

Aisen also says that 18 months may not have been long enough to pick up any clinical effect on the memory or behaviour of patients. "One of the huge difficulties of disease-modifying treatments in general is that they aim to slow the rate of progression of the disease and this is what trials have to measure," he says. With a disease that advances slowly, as Alzheimer's does, there may be only a small difference in how fast it progresses between patients in the treatment group and those in the control group. Furthermore, any difference may take a long time to become apparent. That means that trials need to recruit large numbers of patients and follow them for a long time - and that costs a lot of money.

\section{Early start}

Yet another problem is that disease-modifying drugs are best deployed in the early stages of disease, and it is not currently possible to identify Alzheimer's before dementia sets in. Some of the promising preclinical results are based on mouse models of Alzheimer's that are treated before symptoms develop. By the time patients are recruited into trials, they are already taking such an array of therapies, including memory-enhancing drugs and antidepressants, that variability in both subject and control groups confounds analysis further.

The only way round these problems, everyone agrees, is to develop reliable biomarkers to help follow the progress of disease and treatment. In fact, the search has been on for several years.

There are two main biomarker approaches. One is to image the brain to determine its structure, its activity and how much amyloid it contains. The other involves measuring concentrations of amyloid and tau peptides in the cerebrospinal fluid and perhaps even in the blood. These techniques could be used to select those with true Alzheimer's for clinical trials. They might also help neurologists to follow the disease as it

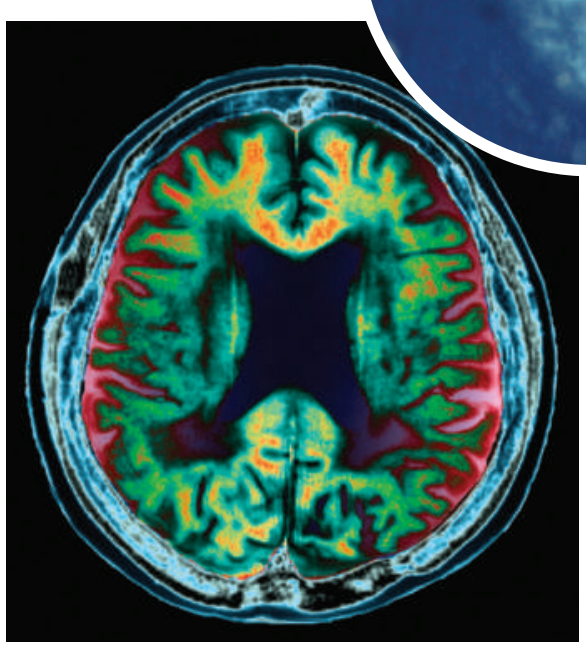

Protein plaques (above) contribute to the destruction of brain tissue in Alzheimer's disease. advances and monitor whether a test drug is having a biological effect (such as removing amyloid) even if the patient has no measurable change in mental state. The major challenge for researchers now is to validate the proposed biomarkers by showing that the detection methods are consistent and reliable in thousands of patients from different clinical centres and that the measurements correlate with biological or clinical endpoints.

The community's perceived need for Alzheimer's biomarkers is reflected in the amount being spent to pursue them. Lovestone is principal investigator of one of the largest consortia of academics and pharmaceutical companies. Known as AddNeuroMed, this $€ 8$.6-million (US\$11-million) five-year scheme is one of only two pilot projects supported by the European Union's $€ 2$-billion Innovative Medicines Initiative, planned to remove research bottlenecks in drug-development processes.

In the United States, Alzheimer's biomarkers are being sought as part of the Biomarkers Consortium launched in October 2006, and by the $\$ 60$-million Alzheimer's Disease Neuroimaging Initiative. The neuroimaging initiative began in 2004 and is following the neuropsychiatric progression of 200 healthy elderly people, 400 patients with mild cognitive impairment and 200 with Alzheimer's disease using various test biomarkers. Both consortia are supported by the National Institutes of Health and by several pharmaceutical companies. These and various other initiatives under way in Japan, Australia and China, coordinate with each other carefully, sharing protocols and data and having regular conference calls, not least because those doing clinical trials are keen for drug regulatory agencies such as the US Food and Drug Administration and the European Medicines Agency to accept their validity.

Magnetic resonance imaging is already widely used to monitor key affected parts of the brain, such as the medial temporal lobe, as they shrink in nearly all patients with Alzheimer's disease, but in fewer than $30 \%$ of the normal ageing population. Changes in brain volume are believed to correlate well with memory performance. Positron-emission tomography (PET) imaging, used to measure the rate of metabolism in different brain areas, can also be used to detect Alzheimer's, although there is large variability between centres. A new PET technique developed by Steven DeKosky from 


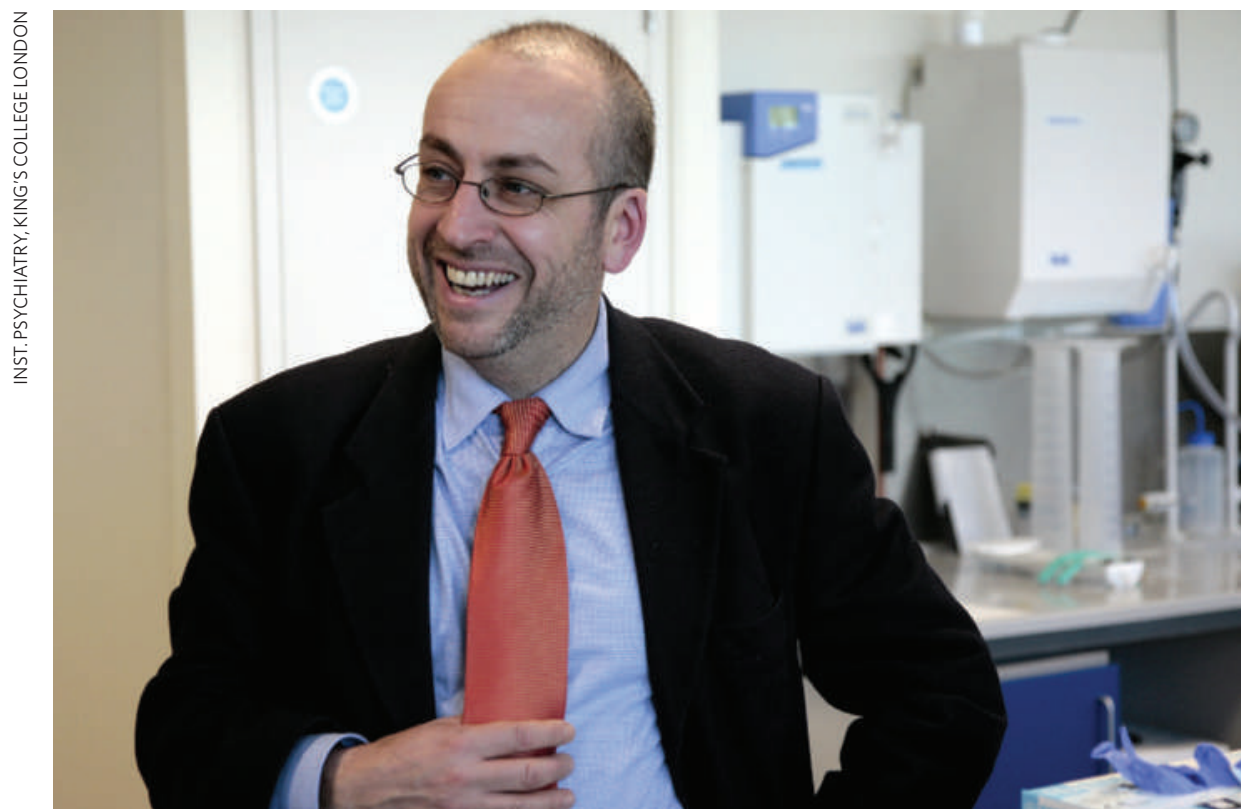

Simon Lovestone wonders whether too much focus has been placed on early parts of the amyloid cascade.

the University of Pittsburgh in Pennsylvania and his colleagues uses special radioactive ligands that bind to amyloid peptides ${ }^{5}$ to visualize plaques directly, allowing them to be tracked as the disease progresses, and as treatment interferes with them.

Concentrations of amyloid peptides, particularly one called amyloid-61-42, are low in the cerebrospinal fluid of patients with Alzheimer's disease compared with healthy controls because the plaques are thought to suck them out of circulation, and concentrations of tau protein and phosphorylated tau are high. The concentrations and ratios of these three proteins can be useful indicators of whether a test drug is having a biological effect in the brain, and are already being used in some of the 30 or so trials that are now under way for treatments based on the amyloid-cascade hypothesis. These include a phase III study by Eli Lilly in Indianopolis, Indiana, of a $\beta$-secretase inhibitor and further studies of bapineuzumab.

None of these methods is as yet sensitive or specific enough to act as a biomarker for Alzheimer's on their own. In a paper published last year ${ }^{6}, 19$ authors representing centres in Europe, North America and Japan, proposed that diagnosis of Alzheimer's should include at least one of the biomarkers under development in addition to new memory assessment techniques that can differentiate more precisely between different dementias. The authors hope that this approach might be applied routinely to diagnose a condition known as 'mild cognitive impairment', which often precedes fullblown Alzheimer's. If it were possible to identify patients with mild cognitive impairment who were highly likely to progress to Alzheimer's, they could form the basis of much more useful cohorts in clinical trials, says Dubois, who was lead author on the paper.

Eventually, and this is the trickiest part, a set of proven biomarkers may be able to act as surrogates for the disease, just as CD4positive cells are used as a surrogate for HIV progression. When antivirals were first tested against HIV, their efficacy was measured based on the number of days a patient spent in hospital, or on time until death, says Michael Weiner, director of the Center for Imaging of Neurodegenerative Diseases at the Veteran Administration Medical Center campus of the
"Biomarkers are really changing our approach to studying and, hopefully, treating the disease." - Dale Schenk especially those performing imaging. "We'll be persuaded when the scientific community gives systematic validation data that are reproducible everywhere," she says. "We are not going to see a surrogate marker for Alzheimer's anytime soon."

Most pharmaceutical companies seem to be unwavering in their pursuit of the amyloid target, with several beginning new trials and few giving up on those that are running. Even though Elan's early-phase bapineuzumab trials did not perform well across the board, post-hoc analysis indicated some improvement in a subgroup of patients who, counterintuitively, did not carry a risk gene for the disease called APOE4. That was more than enough to give the company confidence to carry on, says Dale Schenk, Elan's chief scientific officer. He says that the company has set up four phase III trials, involving some 4,000 patients, of which two trials will evaluate those who carry an APOE4 mutation, and two will evaluate patients without it.

Schenk believes that biomarkers will also make all the difference. Some patients in the bapineuzumab studies are being evaluated with the new PET technique for imaging plaques in living brains. "Biomarkers are really changing our approach to studying and, hopefully, treating the disease," Schenk says. "These technologies are promising in their ability to show us whether amyloid is being cleared from the patient's brain while the treatment is going on."

Some serious attention is now being given to tau as a clinical target. One clinical trial under way by the Singapore-based company TauRx Therapeutics is testing whether methylthioninium, commonly known as methylene blue, can clear tangles. "I believe that eventually we'll find we need different therapeutic strategies University of California, San Francisco. Once CD4 was shown to correlate with clinical outcome, "they didn't have to wait for people to die any more - and this is what we want in Alzheimer's". Surrogate endpoints might allow clinical trials to be shorter and cheaper.

\section{Surrogate scepticism}

But regulatory agencies need a lot of persuasion to accept a new surrogate. In the past year, the validity of even very established biomarkers - such as blood cholesterol for cardiovascular health and blood glucose for diabetes - have been called into question when trials showed they were poor indicators of morbidity or mortality ${ }^{7}$. Marisa Papaluca-Amiti, a biomarkers expert at the European Medicines Agency, says that different centres still show a high level of variability for Alzheimer's biomarkers, for different stages of the disease, including symptomatic treatments as well as diseasetargeted treatments," says Bob Holland, vicepresident for neuroscience at AstraZeneca in Wilmington, Delaware. "But amyloid-targeted therapies are highly like to be a big part of it."

Those with Alzheimer's, and the people caring for them, face difficult tests every day. But the biggest tests of the amyloid hypothesis

Alison Abbott is Nature's senior European correspondent.

1. Ferri, C. P. et al. Lancet $366,2112-2117$ (2005)

2. Holmes, C. et al. Lancet 372, 216-233 (2008)

3. Lippa, C. F., Nee, L. E., Mori, H. \& St George-Hyslop, P. Lancet 352, 1117-1118 (1998).

4. Roberson, E. D. et al. Science 316, 750-754 (2007).

5. Ikonomovic, M. D. et al. Brain 131, 1630-1645 (2008).

6. Dubois, B. et al. Lancet Neurol. 6, 734-746 (2007).

7. Ledford, H. Nature 452, 510-511 (2008). are yet to come. 